\title{
Hk Maker Lab: An Engineering Design Summer Program for High School Students
}

\author{
Aaron M. Kyle', Michael Carapezza', and Christine Kovich², \\ ${ }^{1}$ Columbia University Department of Biomedical Engineering; ' $H Y$ YPOTHEkids, Inc. \\ Keywords: Biomedical Engineering, Engineering Design \\ Publication date: January 21, 2018 \\ DOI: https://doi.org/10.15695/jstem/v1i1.5
}

\begin{abstract}
Increased emphasis on K-12 engineering education, including the advent and incorporation of the NGSS in many curricula, has spurred the need for increased engineering learning opportunities for younger students. This is particularly true for students from underrepresented minority populations or economically disadvantaged schools, who traditionally lag behind their peers in the pursuit of STEM majors or careers. To address this deficit, we have created the Hk Maker Lab, a summer program for New York City high school students that introduces them to biomedical engineering design. The students learn the design process through a series of interactive workshops and laboratory activities. In coordination with learning the process, students apply the engineering design process to identify real-world problems and create testable prototypes. The students explore the entrepreneurial aspects of their projects, creating basic business plans. The program has been successful in creating a diverse set of program participants who have learned engineering design and created solutions to open-ended problems. This paper presents the structure of the Hk Maker Lab and preliminary assessments of the program, providing a basic framework for those similarly interested in creating STEM education opportunities for high school students.
\end{abstract}

\section{INTRODUCTION}

The current deficit in the quality and quantity of K-12 STEM education in the U.S. (National Science Board, 2007; US Department of Education, 2008; Jeffers et al., 2004; Katehi et al., 2009; Kimmel et al., 2007; Project 2061, 1993; Silverstein et al., 2009) is particularly detrimental to underrepresented minority (URM) students who often do not have access to, or elect not to take, STEM courses (Griffith, 2010). One reason for this shortcoming is the rift between STEM education and the practical utility of STEM knowledge. The lack of elementary and secondary STEM education propagates throughout subsequent academic and professional pursuits: African Americans and Hispanics or Latinos account for only $16.1 \%$ of students enrolled in undergraduate engineering degree programs (NSF, 2017). Significantly, even when minority students pursue four-year STEM majors, only $13.2 \%$ of African Americans and $15.9 \%$ of Hispanics complete their STEM degrees (HERI, 2010). This lack of STEM education manifests in minorities not attaining STEM-related occupations: African Americans and Hispanics comprise a combined $11 \%$ of the engineering and science workforce (NSF, 2017).

The Next Generation Science Standards (NGSS), which are being incorporated into an increasing number of K-12 curricula, emphasize engineering and design principles. The National Academy of Engineering and the National Re- search Council Center for Education stress that STEM education should include engineering design training; however, there remains a lack of cohesive engineering thrusts in primary and secondary school curricula (Katehi et al., 2009). This gap misses a huge opportunity, as engineering design presents a number of educational advantages including but not limited to: fostering and enhancing skills for identifying and developing solutions to problems; encouraging students to think critically about important societal challenges; and linking classroom outcomes to tangible artifacts that can promote continued interest in STEM.

To address the pressing need for high school engineering design education, we have created the HYPOTHEkids (Hk) Maker Lab, a summer program that introduces high school students from underserved communities to biomedical engineering (BME) and the engineering design process (EDP). The primary goals of the Hk Maker Lab are to:

1. Increase high school students', particularly those from underrepresented minority groups and economically disadvantaged schools, appreciation for STEM via an emphasis on engineering and the EDP,

2. To have high school students successfully learn and utilize the EDP to identify and address open-ended problems.

The EDP empowers students by providing them with a 
systematic framework for understanding and solving real-world problems. Hk Maker Lab challenges students to apply EDP principles to innovate solutions to problems of their identification. The Hk Maker Lab is meant to be a formative educational experience, encouraging students to continue pursuing opportunities in STEM fields, including undergraduate degrees and careers. The goal of this programmatic paper is to outline the structure of the Hk Maker Lab summer program for the purposes of dissemination and potential utilization of this model by those who strive to create engineering-centric programs. Additionally, program efficacy is demonstrated through preliminary assessment of our participants' responses to attitudinal surveys of their knowledge of engineering and design, experiences in the program, and their plans to pursue STEM undergraduate majors.

\section{PROGRAM STRUCTURE}

The Hk Maker Lab is a six-week summer program in which high school students learn and apply the engineering design process. Participating students are required to complete a comprehensive application and in-person or video interviews to attain program admission. The program is held in classrooms and laboratories at Columbia University - Morningside Campus. Students, typically rising Juniors and Seniors, participate in the program for four days per week from 10 a.m. -4 p.m. The engineering design training is imparted through a combination of workshops, laboratory procedures, and prototyping work.

Engineering design workshops. The Hk Maker Lab workshops are classroom sessions that are used to impart the EDP to participating students. These sessions are highly interactive, with topic lectures interspersed with in-class activities that allow students to practice the EDP. In-class practice sessions are regularly conducted to give students opportunities to apply novel design concepts as they are introduced. Additionally, students deliver weekly mini-presentations on the outcomes of their class efforts. These presentations are critiqued by their peers giving the entire class opportunities to share and refine their understanding of the EDP as they are learning it.

The engineering design process is segmented into three phases: Problem Identification, Solution Conceptualization, and Solution Implementation and Testing (see Table 1 for the general sequence of Hk Maker Lab EDP instruction). Students are first introduced to methods that innovators use to identify unmet needs. They are charged with conducting extensive research on a biomedical problem such that they understand the underlying pathology, the key stakeholders related to the problem and why a solution presents value to them, and the reasons why existing solutions are insufficient. Once a problem is well-defined and vetted, they are encouraged to generate prospective solutions via unconstrained brainstorming. Students use their previous research into the problems and stakeholders to methodically determine which of their ideas are most appropriate. The students are then charged with devising proof of concept experiments to test the feasibility of their solutions. The experiments reference students' prior knowledge of the scientific method; however, instead of simply presenting them with pre-fabricated experimental procedures, they are challenged to formulate their own experimental questions, hypotheses, and methodologies to test the appropriateness of their solutions. As a result of the experiments, they elect whether to proceed with proto-

Table 1. Exploratory Factor Analysis Eigenvalues

\begin{tabular}{llll}
\hline $\begin{array}{l}\text { Engineering Design } \\
\text { Process Stages }\end{array}$ & Week & EDP Workshops & Vital Signs Monitor Laboratory Procedures \\
\hline Identification & Week 1 & $\begin{array}{l}\text { Problem Identification \& Needs } \\
\text { Finding }\end{array}$ & Body Temperature \\
& Week 2 & Design Research \& Inputs & Breathing Rate \\
& Week 3 & Ideation: Brainstorming & Heart Rate \\
& & & Muscle Activity Measurement \\
Conceptualization & & Proof of Concept Testing & \\
\hline Week 4 & Business Planning & \\
\hline
\end{tabular}


Table 1. Exploratory Factor Analysis Eigenvalues

\begin{tabular}{lll}
\hline Vital Sign & Engineering Concepts & Design Challenge \\
\hline Body Temperature & Basic Circuits (resistive voltage divider, thermistors) & Problem Definition \& Need Statement \\
\hline Calibration Curve & \\
\hline Heart Rate & Data Acquisition and Digitization & Problem Definition \& Need Statement \\
\hline $\begin{array}{l}\text { Muscle Activity } \\
\text { (Electromyography) }\end{array}$ & Analog Filtering & Design Research \\
\hline
\end{tabular}

typing or identify new prospective solutions and test for appropriateness. In coordination with creating solutions, they are introduced to entrepreneurial aspects of medical device innovation; as a part of their design efforts, they are charged with developing simple business plans that outline the market associated with their projects and how their solutions provide value to customers.

Laboratory procedures. Many of the Hk Maker Lab participants have limited applied technical knowledge. To instill confidence and develop basic skills, students conduct a set of practical laboratory procedures during the first three weeks of the program. These procedures are well-defined, with students building vital signs monitors to measure body temperature, breathing, heart rate, and muscular activity. Most of the technical content in the procedures pertains to electrical circuitry and microcontroller (Arduino) basics (procedure details are outlined in Table 2). The procedures are not only meant to enhance the students' hands-on skills, but also provide them opportunities for EDP practice, as each lab procedure culminates in a design challenge in which they apply engineering design concepts to the recently-completed lab activity. For example, the completion of the breathing monitor lab coincides with EDP instruction on Problem Definition and Need Statements; accordingly, the students identify problems that would require respiratory monitoring. These challenges complement the lab procedures by giving students' opportunities to practice their burgeoning design skills in the context of simple devices that they create via the labs.

Final projects and prototyping. In coordination with design instruction and laboratory activities, the student teams (3-4 people per team) are challenged to apply the EDP to a problem of their identification. Students explore real-world needs that arise from a variety of sources, including but not limited to: their interests, the outcomes of their research, suggestions from the teaching team, or a pre-established pool of global health technology needs. Rather than assign- ing projects, the Hk Maker Lab encourages students to identify their own problems, reflecting the open-ended nature of engineering design. Additionally, having students select their own projects promotes ownership of their work, excitement to take on new challenges, and investment in devising solutions to the problems.

The students' identification of needs and development of an understanding of the issues associated with those needs occurs nearly in parallel with the EDP workshops. Upon completion of the first three weeks of the program, each team is expected to have identified a need and devised a plan to prototype a solution. They spend the last three weeks of the program building, testing, and refining their designs. This portion of the program is almost exclusively spent in the laboratory. Project teams bring their ideas to fruition with the goal of creating functional prototypes. These prototypes are tested with respect to specifications from their research: prototypes whose performance meet the specifications are considered successful, while those that do not are subject to re-design or optimization.

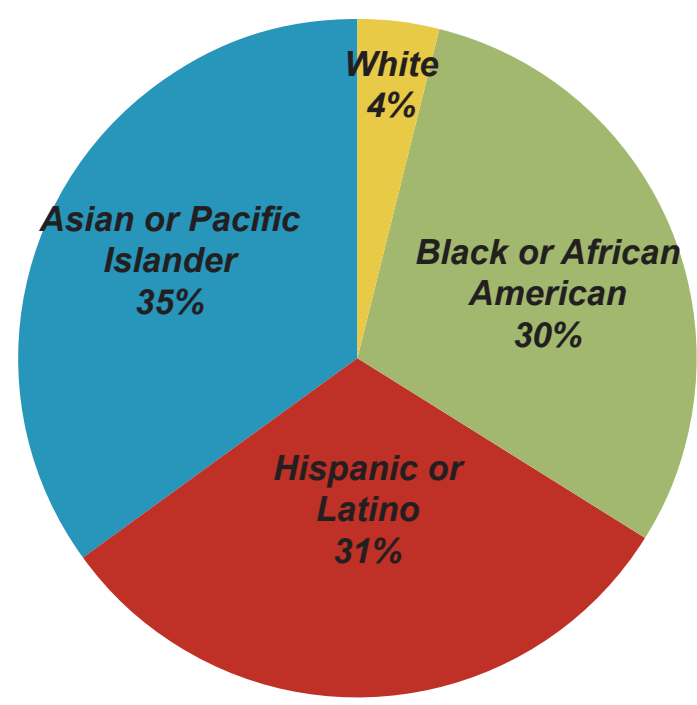

Figure 1. Demographic data for $2017 \mathrm{Hk}$ Maker Lab participants. The 2017 cohort consisted of 22 students, 16 women, and 6 men. 


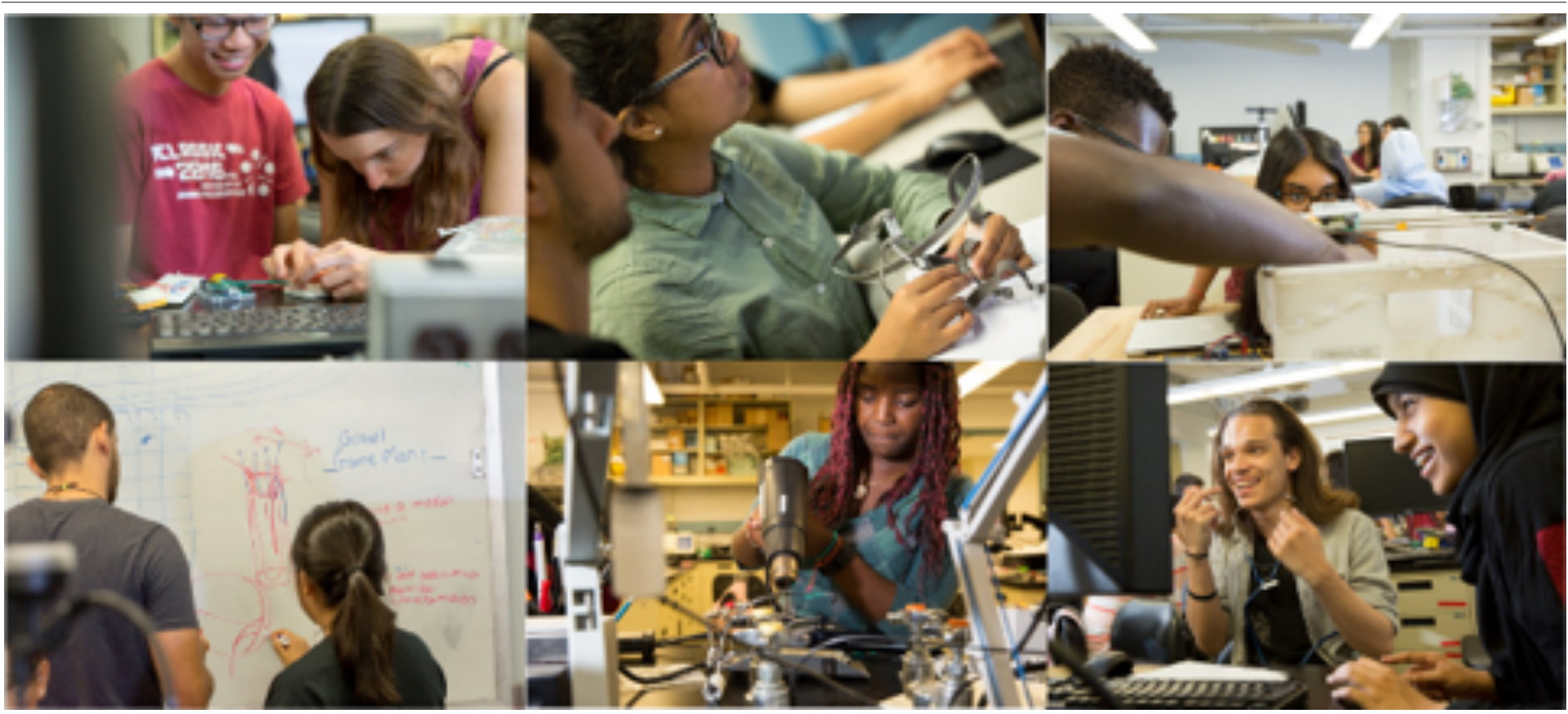

Figure 2. 2017 Hk Maker Lab students developing their project prototypes. Clockwise from top left: an assistive device to maintain proper rate and depth of cardiac compressions during CPR, a mask add-on to prevent the inhalation of airborne pollutants, a non-contact apnea monitor for infants, a device to safely extract a retained placenta, a UV-based needle sterilization device for low resource settings, an automated blood pressure monitor for preeclampsia detection in low resource settings.

\section{PROGRAM OUTCOMES}

The Hk Maker Lab was first offered at Columbia University in the summer of 2014. Since its inception, 94 students have participated in the program. Hk Maker Lab has successfully created a pool of students in which the proportion of underrepresented students exceeds national averages of minorities typically pursuing STEM educational opportunities. The open-ended nature of the problem-identification process has allowed students to explore a wide variety of projects, ranging from a rechargeable battery-powered light array for hospitals in low resource settings to a guidance system to protect the visually impaired from above-the-shoulder obstacles. The student teams have almost always successfully completed their projects: 23 of 24 projects have resulted in prototypes that have satisfied the most critical functional requirements.

The most recent cohort of the Hk Maker Lab (July 17 August 24, 2017) is presented here as a representative example of the program outcomes. This class was comprised of 22 students (Figure 1). Employing their burgeoning expertise in the EDP, these teams created the following biomedical devices (Figure 2):

- Pollution filtration mask to reduce the inhalation of airborne particulate matter;

- Automated blood pressure monitor for detecting pre-eclampsia in pregnant women in low resource settings;

-UV-light sterilization system for preventing secondary needle infections in low resource settings;
- Non-contact apnea monitor for neonates that uses oral and nasal temperature fluctuations to detect breathing;

- CPR adherence device to ensure that chest compressions are administered at the correct frequency and depth;

-Device to safely extract adherent placenta post-delivery.

The Hk Maker Lab culminates in final presentations delivered by student teams in which they describe their biomedical problems and the associated expertise on those problems that they developed throughout the program. They

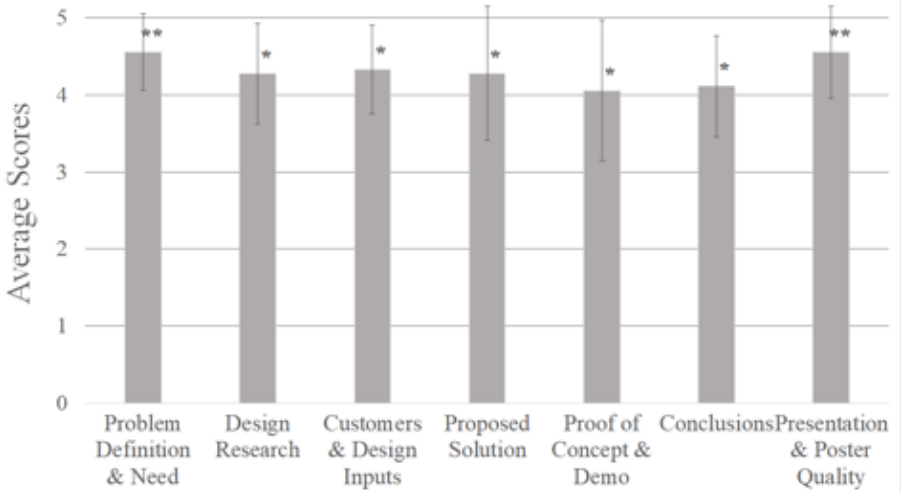

Figure 3. Judges' scores of 2017 Hk Maker Lab projects. Projects were scored using a Likert-type scale $(5 \sim$ Excellent, $4 \sim$ Good, 3 $\sim$ Average, $2 \sim$ Fair, $1 \sim$ Poor) on seven EDP criteria. For each category, scores were averaged across all judges and student teams $(\mathrm{n}=18$ for each category). The mean scores for each team in all of the categories were compared to an average score (3) using a Wilcoxon Signed Rank Test $(\alpha=0.05)$. * indicates a mean score significantly greater than "Average" (3). ** indicates a mean score significantly greater than "Good" (4). 


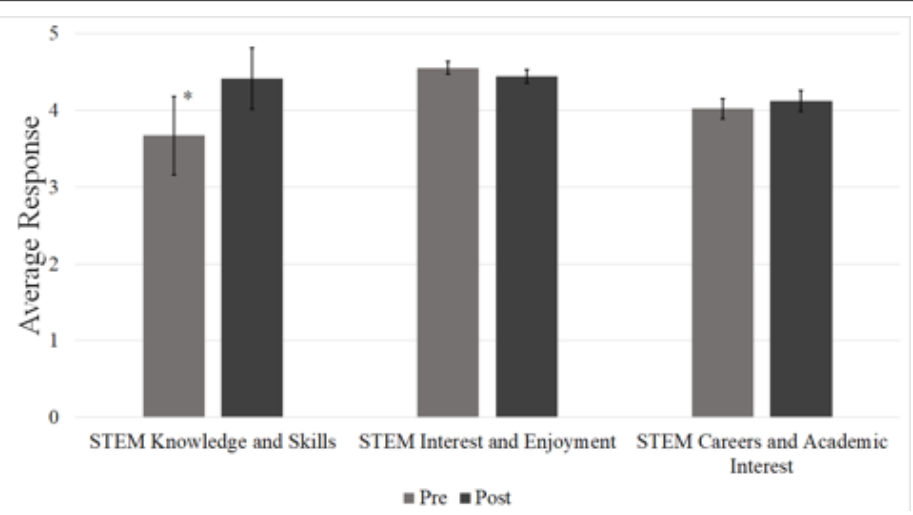

Figure 4. Average pre- and post-program student attitudinal survey responses for the $2017 \mathrm{Hk}$ Maker Lab $(\mathrm{n}=21)$. Students scored their affirmative mastery of concepts on a Likert-type scale (5 Strongly Agree, $4 \sim$ Agree, $3 \sim$ Neutral, $2 \sim$ Disagree, $1 \sim$ Strongly Disagree). The mean pre- and post-course scores were compared using a Wilcoxon Signed Rank Test $(\alpha=0.05)$. * indicates statistically significant difference between pre- and post-survey responses.

extensively discuss their prototyping efforts and testing outcomes. A panel of invited guests from academia, industry, and entrepreneurial sectors evaluates the presentations based on the program objectives and criteria. The judges' feedback determines which team receives the Top Design Award (\$1000 per team) and provides unbiased direct assessment of the students' ability to communicate their needs and project efforts as well as their prototype outcomes. Aggregate judge scoring from the $2017 \mathrm{Hk}$ Maker Lab class is displayed in Figure 3. All mean scores were significantly greater than the "Average" score, indicating that, according to the objective third-party evaluation, our students attained levels of mastery of all of the pertinent design concepts.

Students' knowledge gained from the Hk Maker Lab is indirectly assessed via surveys administered at the start and end of the program. The questions in this survey are designed to provide an overview of student attitudes toward STEM learning and engineering design. The outcomes from the 2017 cohort are indicative that the aforementioned program goals are being met (see Figure 4). Upon entry into the program the students expressed an interest in pursuing STEM majors during their post-secondary studies, and in learning more about engineering design. We did not observe a significant change in these attitudes at the end of the program. We interpret this as a positive finding as the students entered the program with expressed interests in STEM and the EDP that persisted throughout the very demanding and challenging course. Upon entry into the program, the students generally were neutral or not knowledgeable of STEM and engineering skills. But upon completion of the program, students reported an increase in their STEM knowledge attributable to the engineering design experience, supporting the overarching assertion that STEM skills and interest are enhanced as a result of participation in the Hk Maker Lab.

Students from the early offerings of the program of the Hk Maker Lab are beginning their undergraduate matriculation, and, in accordance with our programmatic goals, a large proportion of these students are pursuing STEM or STEM adjacent majors at high quality universities and colleges (Figure 5). All of the underrepresented minorities who have participated in the program are pursuing STEM majors. We have also had four (4) alumni admitted to the program's home institution, Columbia University, and specifically to the School of Engineering and Applied Science. These results indicate that the Hk Maker Lab is positively influencing high school students' early STEM careers.

\section{Project example: Team S.E.A.L.: Airborne pollution} filtration. Team S.E.A.L., a group of three young women engineers, were 2017 Hk Maker Lab participants. When identifying needs, they were inspired by members' visits to Southeast Asian countries and the increasingly prevalent issue of air pollution. They found that that the use of surgical masks, a common method of personal protection from pollution, is ineffective. They learned the specific failures of surgical masks are due to their inability to filter small airborne particulates and that the masks often do not fit, providing unobstructed points of entry for pollutants. To address this need, the group brainstormed a re-designed high efficiency filtration mask that could be custom-fit to a wearer's face. Their mask would use a moldable plastic rim to allow the user to shape it to their face, ensuring a secure fit, and would have a compartment in front of the mouth in which a disposable filtration membrane could be inserted each day.

Initial proof of concept testing successfully demonstrated that the contoured plastic rim would create an impermeable seal to pollutants. The team tested numerous filter materials,

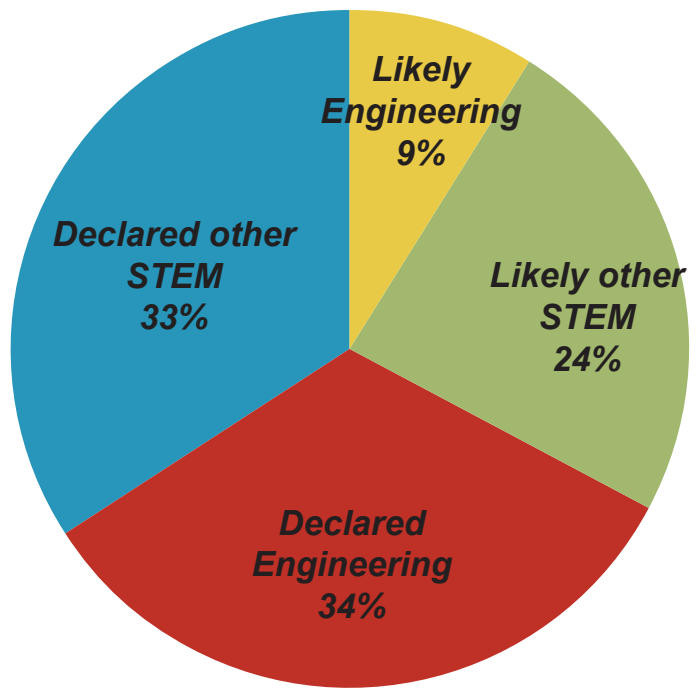

Figure 5. Hk Maker Lab alumni college majors $(n=33)$. "Other STEM" here refers to any non-engineering STEM major. No (0) respondents indicated that they intended to pursue non-STEM majors. 


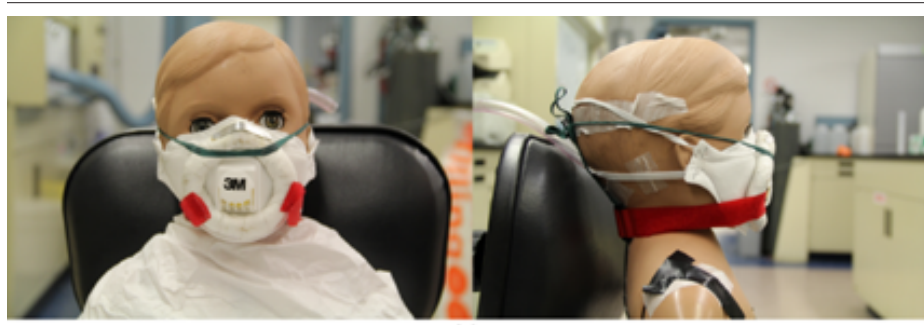

(a)

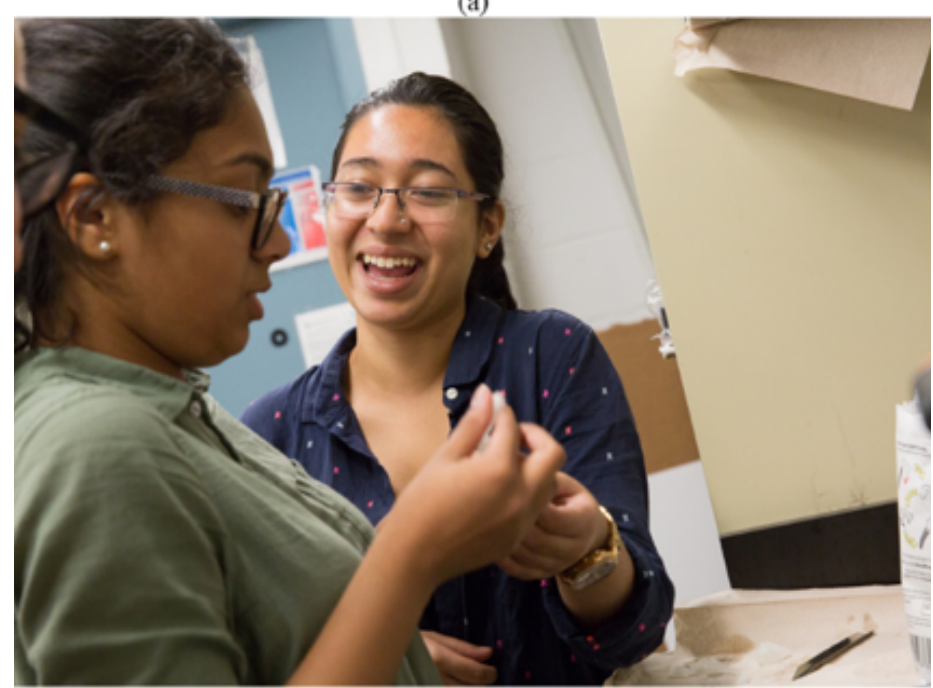

(b)

Figure 6. Images of $2017 \mathrm{Hk}$ Maker Lab Team S.E.AL. (a) The moldable seal coupled with the filtration mask and (b) team members developing their test bed to the demonstrate efficacy of the seal.

but they were all either poor barriers or so impermeable that one could not breathe through them. Rather than accepting failure, the group embraced that their initial filtering concept would not address the problem and decided to pivot. They found that there are existing masks that provide adequate filtration; however, these masks are still susceptible to the poor fit issues that are problematic with surgical masks. They decided to take their successful moldable plastic rim and turn it into a device that would complement the filtering component of these masks - their reusable, moldable plastic rim would be coupled with the masks to seal them to the face, forcing inhaled air to pass through the filter. With their revised solution, the team created an appropriate test bed, using a human CPR dummy to model breathing and simulating pollution exposure by flowing incense smoke (which was found to be a reasonable simulacrum for airborne pollution) through the model. Video analysis of their tests showed that their moldable mask rim prevented pollutants (smoke) from traversing the mask compared to the mask alone (see Figure 6).

Team S.E.A.L. excellently embodied the critical lessons of the Hk Maker Lab. They independently identified a healthcare need that aligned with their interests and experiences. They devised a solution based on the problem as well as customer needs. Initial testing demonstrated that aspects of their solution, i.e., the moldable seal, would address a shortcoming of existing masks, while their proposed filter- ing initially appeared not applicable. Rather than discarding the problem and solution, the team embraced the spirit of iterative re-design, using their previous findings to create version of their device that would address their problem. Their battery of tests allowed them to quantitatively prove that their mask system was better than existing solutions. Team S.E.A.L. demonstrated the ability of young students to employ the EDP.

\section{CONCLUSIONS AND FUTURE WORK}

We aspire to translate the Hk Maker Lab educational model to other institutions that are interested in offering similar pre-college programs to URM and economically-disadvantage students. We intend to freely distribute course materials, findings, and outcomes of our research related to this endeavor. We emphasize design as a powerful paradigm for applied STEM; however, we propose that emergent programs utilizing our model need not be constrained to engineering design. The approach of instruction via interactive workshops, lab procedures for skills development, and an overarching project can be employed in the context of a variety of disciplines. Regardless of the program focus, it is critical that the lead instructor has significant expertise in the topic area coupled with a passion for imparting the concepts to students. The questions and needs of the high school students are quite different from undergraduate or graduate students, especially in the context of an open-ended project program. High school students express significant discomfiture with ill-defined projects, so they require a great deal of guidance in applying pre-existing and novel knowledge.

In addition to instructor expertise, it is highly encouraged that, dependent on the size of the class, educational support staff be employed. The Hk Maker Lab employs a cohort of graduate and undergraduate teaching assistants (TAs) throughout the program. The TAs provide instructional support along with near-peer mentorship to the Hk Maker Lab participants. During the prototyping phase of the program, there is at least one TA for each project team, ensuring that the teams have the requisite guidance as they are developing their solutions. Additionally, these types of projects require a well-appointed work space, complete with the necessary supplies and equipment that will allow for rapid preliminary experiments or prototyping efforts. A proper workspace facilitates physical realization of ideas shortly after they are devised, minimizing lag times that might be experienced if parts or equipment have to be procured on an as-needed basis.

The Hk Maker Lab is one component of a larger suite of activities meant to enhance engineering knowledge and pursuit of STEM in underrepresented minority and economically disadvantaged high school students. As a result of the summer program, we have created internships that provide Hk Maker Lab alumni with opportunities to further explore 
biotechnology careers. Over the past three years, we have placed 24 of our alumni in high quality internships in research laboratories throughout the School of Engineering and Applied Science at Columbia University and with NYCbased biotechnology startup companies. These internships are meant to further engender the students' interest in the pursuit of STEM by exposing them to the careers that can arise from science and engineering-centric education.

Another expansion of the Hk Maker Lab is the creation of engineering design-centric modules and/or courses for high schools. The summer program is constrained by the size of our workspaces and instructional team to 24 students per year. However, it is increasingly critical that all high school students have engineering, particularly design, opportunities in their high school courses. To address this need, we are working with local high school engineering, science and math teachers to develop engineering design curricular modules that can be used in their respective schools for in-classroom EDP instruction. The resultant courses and content will be rigorously evaluated and optimized. Similar to the summer program, we intend to distribute the curricula and the findings from this endeavor to support increased engineering design education for high school students, increasing the population of students who will have their STEM interests complemented by innovation education.

We have successfully created a summer program in which high school students are introduced to engineering design and apply it to uncover and solve biomedical problems. The Hk Maker Lab emphasizes working with students from underrepresented minority groups and economically disadvantaged schools from throughout New York City, giving these young people opportunities that they might not normally receive in their typical educational experiences. As evidenced by their successful project outcomes, the students are highly receptive to the engineering design education and able to readily apply their newfound knowledge to real-world problems. We assert that the emphasis on problem identification and solving gives the students an increased appreciation of applied science and engineering that will motivate them to further explore STEM education and careers.

\section{AUTHOR INFORMATION Corresponding Author}

Aaron M. Kyle, Ph.D., Columbia University, Department of Biomedical Engineering, ak3110@columbia.edu

\section{Author Contributions}

The manuscript was written through contributions of all authors. All authors have given approval to the final version of the manuscript.

\section{FUNDING SOURCES}

This work was supported by the National Institutes of Health Science Education Partnership Award (R25OD021905-02) and a grant from the Pinkerton Foundation..

\section{ACKNOWLEDGMENTS}

The authors would like to thank our assessment partners, Education Development Center - Children's Center for Technology.

\section{ABBREVIATIONS}

STEM: science, technology, engineering and mathematics; URM: underrepresented minority; NGSS: Next Generation Science Standards; HK: HYPOTHEkids; EDP: engineering design process; BME: biomedical engineering

\section{REFERENCES}

Griffith, A. (2010). Persistence of women and minorities in STEM field majors: Is it the school that matters? Economics of Education Review, 29, 911-922.

Higher Education Research Institute at UCLA. (2010). Degrees of Success: Bachelor's Degree Completion Rates among Initial STEM Majors, HERI Report Brief.

Jeffers, A., A. Safferman, and S. Safferman. (2004) Understanding K-12 engineering outreach programs. Journal of Professional Issues in Engineering Education, 130, 95-108.

Katehi, L., Pearson, G., Feder, M. A., and Committee on K-12 Engineering Education. (2009). Engineering in K-12 education: Understanding the status and improving the prospects. Washington, DC: National Academies Press.

Kimmel, H., J. Carpinelli, and R. Rockland. (2007). Bringing Engineering into K-12 Schools: A problem looking for solutions. International Conference on Engineering Education. Coimbra, Portugal.

National Science Foundation, National Center for Science and Engineering Statistics. (2017). Women, Minorities, and Persons with Disabilities in Science and Engineering: 2017. Special Report NSF 17-310. Arlington, VA. Retrieved from https:/www.nsf.gov/pstatistics/2017/nsf17310/data. $\mathrm{cfm}$

National Science Board. (2007). National Action Plan for Addressing the Critical Needs of the U.S. Science, Technology, Education, and Mathematics Education System.

Office of Elementary and Secondary Education, U.S. Department of Education. (2008). Mathematics and science partnerships.

Project 2061 (American Association for the Advancement of Science). (1993). Benchmarks for science literacy. New York: Oxford University Press. xv, 418. 
Silverstein, S., Dubner, J.. Miller, J., Glied, S., and Loike, J.D., (2009). Teachers' participation in research programs improves their students' achievement in science. Science, 326(5951), 440-442. 